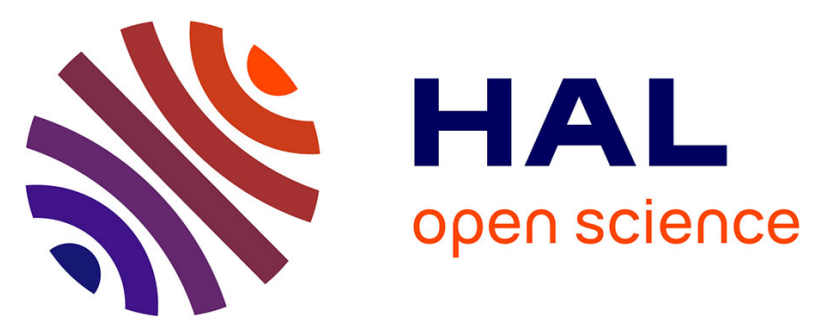

\title{
Crowdequity et Crowdlending: deux alternatives de financement des TPE/PME. Le cas de l'entreprise Gifts for change
}

\author{
Malek Hamouda, Imen Mejri, Donia Trabelsi
}

\section{- To cite this version:}

Malek Hamouda, Imen Mejri, Donia Trabelsi. Crowdequity et Crowdlending: deux alternatives de financement des TPE/PME. Le cas de l'entreprise Gifts for change. 1ère journée d'étude sur le crowdfunding "Crowdlending et crowdequity en France - prospective", Jun 2017, Paris, France. hal02373759

\section{HAL Id: hal-02373759 \\ https://hal.science/hal-02373759}

Submitted on 21 Nov 2019

HAL is a multi-disciplinary open access archive for the deposit and dissemination of scientific research documents, whether they are published or not. The documents may come from teaching and research institutions in France or abroad, or from public or private research centers.
L'archive ouverte pluridisciplinaire HAL, est destinée au dépôt et à la diffusion de documents scientifiques de niveau recherche, publiés ou non, émanant des établissements d'enseignement et de recherche français ou étrangers, des laboratoires publics ou privés. 


\title{
Crowdequity et Crowdlending : deux alternatives de financement des TPE/PME Le cas de l'entreprise Gifts for change
}

\author{
Malek HAMOUDA - ISERAM - ISG Programme Business \& Management \\ Imen MEJRI - NEOMA Business School \\ Donia TRABELSI - Telecom Ecole de management - IMT
}

\section{Résumé}

Le crowdfunding rémunéré s'est fortement développé ces dernières années. II se positionne comme une solution alternative à la problématique de financement des PME/TPE. Notre recherche étudie les atouts et les limites du financement participatif rémunéré comparé au financement traditionnel et met en évidence les spécificités du crowdlending et de l'equity crowdfunding. Notre étude porte sur l'entreprise Gifts for Change, qui a la particularité d'avoir été financée par ces deux types de financement participatif spéculatif. L'analyse montre que le crowdfunding rémunéré dispose de différents dispositifs qui ne sont adaptés qu'à certains projets. Aussi, le choix entre le crowdlending et l'equity crowdfunding ne se pose pas au même stade de développement de l'entreprise. Ce travail permet également d'émettre les principales recommandations à adresser aux entrepreneurs souhaitant se financer via le crowdfunding rémunéré.

Mots clés: Crowdfunding rémunéré; Equity crowdfunding ; Crowdlending ; Atouts ; Limites. 


\section{Introduction}

Les TPE/PME rencontrent d'importantes difficultés pour se financer auprès des piliers du financement classique. Ce financement traditionnel est effectivement de moins en moins accessible à cause d'un contexte de crise économique et des nouvelles réglementations restrictives. Pour répondre aux besoins des entrepreneurs, de nouveaux produits de financement ont vu le jour sur le marché à savoir le financement participatif ou le crowdfunding qui se base sur la puissance de l'internet afin d'assurer une proximité entre les bailleurs de fonds et les entrepreneurs (Agrawal et al., 2011 ; Mollick, 2014).

II existe plusieurs catégories de crowdfunding. La forme la plus médiatisée est le don, avec ou sans contrepartie (Mollick, 2014 ; Vulcan, 2016). Cependant, nous assistons depuis quelques années à l'émergence de plateformes de financement participatif rémunéré. Dans ce cadre, nous distinguons le financement participatif par souscription d'actions (equity crowdfunding) et le financement participatif sous forme de prêt (crowdlending). Le développement de ces deux composantes du crowdfunding a été important. Selon le baromètre du crowdfunding du 21 février $2017^{1}$, les levées de fonds réalisées en France par les plateformes de prêt ont augmenté de $46 \%$ entre 2015 et 2016. Cette évolution est de 36\% pour les plateformes d'investissement en capital, pour la même période. II convient de préciser que l'equity crowdfunding a effectué sa plus forte croissance en 2014 $(+146 \%)$. Quant au financement sous forme de prêt, il a enregistré l'évolution la plus importante en 2015 (+122\%). En parallèle, les limites du crowdfunding et les risques sous-jacents sont de plus en plus mis en avant. Par exemple, en France, le service des études de l'association des consommateurs QFC-Que Choisir a étudié 337 entreprises ayant eu recours au financement participatif ainsi que les plateformes auxquelles elles ont fait appel. Le rapport publié le 23 février $2017^{2}$ met en garde contre les risques du secteur et contre les abus de certains de ses acteurs. On y dénonce «la promesse de rendements surestimés, la présentation partiale et la sélection hasardeuse des projets à financer, des obligations légales insuffisamment respectées ainsi qu'une multitude de clauses abusives au sein des conditions générales d'utilisation des plateformes... ».

Le développement du crowdfunding dans le paysage financier français et l'intérêt croissant des entreprises pour le crowdfunding spéculatif, malgré ses risques éventuels, posent la question de la pertinence de cette alternative de financement. Ainsi, nous nous proposons dans cet article de mettre en exergue les deux composantes du crowdfunding rémunéré : le financement participatif par souscription d'actions et le financement participatif sous forme de prêt. Plus précisément, nous tentons de répondre aux questions de recherche suivantes : pourquoi un entrepreneur ferait-il appel au financement participatif rémunéré plutôt qu'au

\footnotetext{
1 Baromètres 2013, 2014 et 2015 réalisés par CompinnoV pour Financement Participatif France (FPF), l'association des professionnels du crowdfunding, et baromètre du 21 février 2017 réalisé par Financement Participatif France (FPF) et KPMG.

2 «Les placements participatifs: Des placements risqués au potentiel moindre que le livret $A$ » disponible sur www.quechoisir.org/action-ufc-que-choisir-financement-participatif-alerte-sur-lesrisques-et-abus-n24530/.
} 
financement classique ? Et dans ce cadre, quels sont les spécificités du crowdlending et de l'equity crowdfunding?

Notre article présente une étude qualitative exploratoire à travers l'analyse de l'entreprise Gifts for change (Treez) qui a effectué deux levées de fonds via le financement participatif rémunéré : la première sous forme de prêt auprès de la plateforme Blue Bees et la deuxième sous forme de participation en capital auprès de la plateforme Wiseed. Dans ce cadre, nous nous basons notamment sur l'analyse d'entretiens semi-directifs effectués avec le fondateur de cette entreprise mais également avec des responsables au sein des deux plateformes. Ce cas est intéressant car il nous permettra d'effectuer une analyse comparative entre le crowdlending et le crowdequity. À notre connaissance, notre étude empirique est la première étude qui a l'ambition de comparer les deux formes du financement participatif rémunéré.

Le papier s'organise comme suit : d'abord, nous présentons le contexte dans lequel s'est développé le financement participatif rémunéré. Ensuite, nous abordons les atouts et les limites du crowdfunding rémunéré par rapport au financement conventionnel. Les spécificités du crowdlending et de l'equity crowdfunding, sont mises en exergue. Enfin, nous exposons notre méthodologie et présentons notre analyse avant de conclure.

\section{Un contexte favorable au développement du financement participatif rémunéré}

Le crowdfunding rémunéré a vu le jour à la fin des années 2000. En France, ce mode de financement s'est développé dans un contexte caractérisé par une conjonction de facteurs: des facteurs structurels liés à l'évolution du secteur de financement traditionnel (banques et fonds de capital investissement) et des facteurs spécifiques liés à l'émergence du secteur du financement participatif lui-même.

D'abord, nous enregistrons un ralentissement du crédit bancaire aux PME en France (Kremp et Piot, 2014). Les TPE et les PME s'adressent naturellement aux banques quand elles ont besoin de se financer en les considérant comme un acteur privilégié du financement notamment à cause de l'accès limité aux marchés financiers (Bendriss et al., 2014). Cependant, les deux dernières crises financières (la crise des subprimes en 2008 et la crise de la dette souveraine en 2011), ainsi que la mise en place progressive de la nouvelle règlementation prudentielle post-crise (Bâle III) ont changé la donne. Aujourd'hui, bien que les entreprises françaises ne semblent pas avoir connu une situation de rationnement de crédit («credit crunch ») ${ }^{3}$, nous notons une atonie de la distribution du crédit et une diminution de la dette bancaire en particulier la part dédiée au financement de l'exploitation (Kremp et Piot, 2014). Les auteurs, expliquent cette tendance par la baisse de la demande des entreprises. Cependant, ils précisent que "les PME de très petite taille ou de création récente ont de vraies difficultés d'accès au crédit bancaire ».

Ensuite, les entreprises, en particulier au stade de démarrage, ont de plus en plus de mal à se financer auprès des fonds de capital investissement, spécialistes du

\footnotetext{
${ }^{3}$ D'après l'enquête de la BCE et de la Commission Européenne auprès des PME européennes (2013) et le rapport de l'Insee, « Les entreprises en France », 2014.
} 
financement des jeunes entreprises non cotées. Le private equity a été fortement touché par la dernière crise économique et financière (Mahieux, 2012). À cette période, les montants investis par les fonds de capital-risque et de capital-amorçage dans les start-ups et les entreprises innovantes françaises ont enregistré une baisse après des années de forte croissance ${ }^{4}$. À présent, malgré l'assainissement du secteur du capital investissement, sa sélectivité est de plus en plus accrue et la présence des business angels est encore insuffisante (Kettani et Villemeur, 2012). Ainsi, les projets au stade d'amorçage, à cause du risque élevé et de l'asymétrie d'information, rencontrent des difficultés à se financer par les acteurs qui leur sont $a$ priori destinés.

Enfin, une nouvelle catégorie d'investisseurs a émergé sur les marchés. En effet, de plus en plus d'investisseurs souhaitent reprendre le contrôle de leur épargne et aspirent à participer directement à l'économie réelle (Lesur, 2016). Le développement de l'internet participatif ou social à travers la notion du web 2.0 ou le web collaboratif (O'Reilly, 2005) a permis de mettre en relation cette nouvelle catégorie d'investisseurs avec les porteurs de projets à la recherche de financement (Agrawal et al., 2011). La logique financière du crowdfunding où les contributeurs cherchent à réaliser un gain sous forme d'intérêts ou de plus-values en capital s'est progressivement installée en parallèle à la logique de volontariat, relative au financement participatif sous forme de dons avec ou sans contrepartie (Bertrand et Jakubowski, 2016).

Dans ce contexte, un nouveau cadre réglementaire qui se veut sécurisant pour les contributeurs, tout en étant allégé pour les plateformes, a été mis en place. En France, les pouvoirs publics ont précisé les nouvelles règles de l'exercice de cette nouvelle activité ${ }^{5}$. Les plateformes de crowdfunding spéculatif doivent être immatriculées au registre unique des intermédiaires en assurance, banque et finance tenu par I'ORIAS 6 . Nous distinguons les CIP (Conseillers en Investissement Participatif : plateformes de financement participatif par souscriptions de titres financiers) des IFP (Intermédiaires en Financement Participatif : plateformes de financement participatif sous la forme d'un prêt). II convient de signaler que les plateformes de financement participatif sous forme de dons n'ont pas l'obligation de s'immatriculer auprès de l'ORIAS. Elles peuvent toutefois choisir de s'immatriculer en tant qu'IFP. Selon Fliche et al. (2016), le nouveau cadre législatif permettrait de protéger les investisseurs mais également de réduire les contraintes propres à chaque type de financement participatif spéculatif (prêt et souscription d'actions). Un label a même été créé pour identifier les plateformes qui respectent ces nouvelles règles.

\footnotetext{
${ }^{4}$ Selon les statistiques de l'Association Française des Investisseurs en Capital (AFIC) en 2008.

${ }^{5}$ Ordonnance $\mathrm{n}^{\circ}$ 2014-559 du 30 mai 2014 relative au financement participatif et son décret d'application $n^{\circ}$ 2014-1053 du 16 septembre 2014.

${ }^{6}$ II s'agit d'une association, sous tutelle de la Direction du Trésor, qui a été créée en 2007 pour homologuer les intermédiaires en assurance, conformément à une directive de l'Union Européenne datant de 2002. Depuis le 1er octobre 2014, L'ORIAS a pour mission la tenue et la mise à jour du Registre des intermédiaires en assurance, banque et finance.
} 


\section{Les atouts et les limites du crowdfunding rémunéré, comparé au financement traditionnel}

Le crowdfunding rémunéré partage certains atouts et limites du financement participatif sous forme de dons avec ou sans contrepartie. Toutefois, le crowdlending et l'equity crowdfunding, se distinguent par des avantages et des inconvénients qui leur sont propres.

\subsection{Atouts et limites communs avec le crowdfunding sous forme de dons}

Au regard du contexte dans lequel le crowdfunding a vu le jour et s'est développé, ce nouveau mode de financement se positionne comme un moyen alternatif de financer les TPE et les PME (Fasshauer, 2016). La plateforme offre ainsi aux entrepreneurs la possibilité d'accéder à des ressources financières sans avoir recours aux acteurs établis du secteur financier. Le crowdfunding permet, en effet, le financement direct des projets entrepreneuriaux grâce aux plateformes qui facilitent la rencontre entre l'offre et la demande et garantissent l'interaction directe entre les investisseurs et les porteurs de projets (Giudici et al., 2012). Comparé au financement classique, cette solution de financement présente différents avantages (Golić, 2013 ; Lambert et Schwienbacher, 2010). Dans ce cadre, la distance géographique ne représente plus un frein au financement (Agrawal et al., 2011 ; Belleflamme et al., 2013 ; Valanciene et al., 2013). Aussi, les plateformes permettent de mobiliser des investisseurs qui adhèrent aux mêmes valeurs que l'entrepreneur et qui appartiennent à une même communauté (Fasshauer, 2016). Ces investisseurs supportent un risque moindre puisque l'apport de chacun et donc le risque sous-jacent, sont faibles. Au-delà de la levée des fonds, le financement participatif est un moyen de développer l'activité de l'entreprise à travers le processus de la collecte des fonds (Lambert et Schwienbacher, 2010). Les investisseurs sont des clients potentiels et des ambassadeurs des projets (Mollick, 2014). Ce rôle d'ambassadeurs est particulièrement important dans le cadre du crowdfunding spéculatif puisque la rémunération des investisseurs est conditionnée par le succès du projet. En outre, le financement par crowdfunding permet à l'entrepreneur d'avoir des signaux crédibles en ce qui concerne le potentiel du produit sur le marché (Gerber et al., 2013 ; Valanciene et al., 2013). II offre également un avantage concurrentiel et garantit une promotion de l'entreprise et du projet (Gajda et Walton, 2013). II en résulte une réduction des coûts de conception et de commercialisation et une optimisation du processus de développement de nouveaux produits (Lambert et Schwienbacher, 2010). Gerber et al. (2012) et Belleflamme et al., (2013) s'accordent à dire que les principales raisons pour lesquelles les entrepreneurs font appel au crowdfunding sont la collecte des fonds, les motivations commerciales et marketing, ainsi que le développement de relations et de réseaux.

Toutefois, malgré les avantages mentionnés précédemment, le crowdfunding peut avoir des limites. La littérature sur les inconvénients du financement participatif n'est pas avancée à cause de la jeunesse du secteur et du manque du recul. Les premiers arguments dans ce cadre sont l'absence d'évaluation par les spécialistes et la possibilité de manipuler les donateurs par un discours marketing et des promesses sans fondement (Blanchard et Sabuncu, 2016). Belanovà (2015) énonce un certain nombre d'inconvénients du crowdfunding. D'abord, ce type de financement suit dans la majorité des cas un modèle du "tout ou rien" ; ainsi si le montant demandé n'est 
pas atteint les fonds collectés sont reversés aux contributeurs. De plus, il ne faut pas négliger la pression subie par les entrepreneurs notamment en termes de délai du lancement de l'activité. Par ailleurs, un échec de la campagne peut constituer un frein au développement futur des activités de l'entreprise en question.

\subsection{Spécificités du crowdlending et de l'equity crowdfunding}

Chacune des deux composantes du financement participatif rémunéré, le crowdlending et l'equity crowdfunding, représente des spécificités qu'il convient d'analyser.

Concernant le crowdlending, il présente certains avantages par rapport au crédit bancaire. D'abord, lever des fonds via les plateformes permet à l'entrepreneur d'accéder à l'emprunt sans garanties en contrepartie (Yum et al. 2012). Ensuite, les coûts opérationnels sont réduits et par conséquent les prêteurs peuvent avoir un retour sur investissement plus intéressant (Yum et al. 2012). Enfin, internet et les réseaux sociaux facilitent la communication et la transmission d'informations entre l'emprunteur et les prêteurs (Maier, 2016), accélérant ainsi le processus de levée de fonds comparativement au crédit bancaire traditionnel (Lesur, 2015).

Toutefois, comme le soulignent Cai et al. (2016), l'un des principaux problèmes du crowdlending est l'asymétrie d'information. Selon Herzenstein et al. (2011) et Yum et al. (2012), ce problème peut être en partie réduit si l'emprunteur divulgue des informations personnelles et financières, permettant de rassurer les financeurs et de développer une confiance mutuelle. Ainsi, le succès d'une campagne de levée de fonds via le crowdlending dépend fortement des informations communiquées. Larrimore et al. (2011) stipulent que l'utilisation de récits étendus, de descriptions concrètes du projet et de mots quantitatifs ont un potentiel informationnel et persuasif plus important et augmente la probabilité de succès du financement. Plusieurs auteurs stipulent que la quantité et la qualité des informations sur les projets communiquées par les plateformes favorisent leur financement et le gain de confiance des prêteurs (Greiner et Wang, 2010 ; Herzenstein et al., 2011; Gonzalez et Loureiro, 2014). En outre, afin de réduire l'asymétrie d'information, certaines plateformes de prêt établissent des notations de crédit pour chaque projet. Emekter et al. (2015) montrent que le taux d'échec des campagnes de levée de fonds est négativement lié à la notation financière et positivement lié à la durée de la campagne. Seulement, malgré l'évaluation du risque de défaut de l'entreprise par les plateformes, la sélection finale des projets revient à la foule ce qui représente l'un des principaux risques émanant du crowdlending. Ce processus n'est plus la responsabilité des institutions financières, augmentant ainsi le risque de l'emprunteur et donc celui supporté par le prêteur (Yum et al., 2012).

S'agissant de l'equity crowdfunding, il permet à la foule via une plateforme en ligne, de prendre une participation dans le capital des entreprises de la même manière que les fonds de capital-risque. Comparé aux autres formes de crowdfunding, il permet de lever des montants plus importants (Fausshauer, 2016) et en augmentation constante au fil du temps (Vulkan et al., 2016). L'equity crowdfunding affiche l'objectif clair des contributeurs d'obtenir un rendement monétaire positif sur leur investissement. Cette forme de financement participatif implique l'existence d'une évaluation «pré-money » de chacun des projets (Vulkan et al., 2016) ce qui permet de mettre en avant le potentiel intrinsèque de l'entreprise. 
L'equity crowdfunding palie à la principale limite du capital-risque, sa sélectivité élevée. II représente ainsi une source supplémentaire de capital d'amorçage et s'intègre dans les phases les plus en amont du cycle de financement de l'entreprise (Bessière et Stéphany, 2014). Il peut même être considéré comme une menace pour le venture capital ainsi que les Business angels (Vulkan et al., 2016). Ces derniers apportent certes un soutien financier direct aux projets entrepreneuriaux, mais il n'existe pas de structure reliant les entrepreneurs et les investisseurs (Giudici et al., 2012). Par ailleurs, le nombre d'investisseurs et l'effet «foule " sur les plateformes d'equity crowdfunding permettent de combler le processus de due diligence habituel du private equity (Vulkan et al., 2016). La plateforme renforce le processus d'évaluation en apportant une expertise financière permettant de fixer le prix offert (Bessière et Stéphany, 2014). Girard et Deffains-Crapsky (2016) stipulent que les plateformes de crowdequity peuvent consolider leur dispositif sélectif à travers un montant élevé du ticket d'entrée et la syndication avec des investisseurs initiés (business angels et capital-risqueurs). Cependant, les investisseurs et les plateformes ne jouent pas pleinement le rôle actif que nous retrouvons dans le capital risque et auprès des business angels (Bessière et Stéphany, 2015). Le management de la participation après la levée de fonds paraît difficile à cause du nombre important des investisseurs et de leur faible part dans le capital et de l'accompagnement limité de la plateforme après la clôture de la campagne (Andrieu et Groh, 2013; Fasshauer, 2016). Certes certaines plateformes d'equity crowdfunding adoptent un mode de gouvernance par holding permettant de structurer les interactions entre le porteur de projet et les actionnaires dans la phase post-investissement (Bessière et Stéphany, 2015 ; Girard et Deffains-Crapsky, 2016). Cependant, souvent le holding se limite à une fonction administrative (Bessière et Stéphany, 2015 ; Girard et Deffains-Crapsky, 2016).

Enfin, pour les deux formes de financement participatif rémunéré, le cadre législatif gagnerait à être réajusté notamment dans un objectif d'harmonisation européenne. Fliche et al. (2016) soulignent que ce dernier ne semble pas couvrir l'ensemble des nouvelles formes de crowdfunding qui se développent aujourd'hui. Par exemple, ce cadre règlementaire n'est pas adapté aux plateformes d'equity crowdfunding spécialisées dans le secteur immobilier à cause des instruments financiers utilisés et de la forme juridique de certaines sociétés immobilières. En général, l'utilisation des bons de caisse ou des obligations permet de contourner les contraintes pesant sur les prêts proposés par les IFP. II en résulte un risque plus important pour les investisseurs.

\section{Présentation du cas de l'entreprise Gifts For change (Treez) et méthodologie}

Notre étude empirique adopte une démarche qualitative exploratoire et se base sur une étude de cas. Elle se veut à la fois explicative et descriptive (selon les définitions de Yin 1993 et 2014) et instrumentale (selon la définition de Stake, 1995). Son objectif n'est pas de généraliser nos résultats mais de mettre en évidence des phénomènes particuliers (Yin, 2014).

L'étude porte sur l'entreprise Gifts for change (Treez). Gifts for change est une société qui se définit aujourd'hui comme révolutionnaire dans le secteur de la 
communication par l'objet. Son métier consiste en la conception et la distribution d'objets promotionnels et accessoires de mode à impacts positifs. Elle a été créée en 2014 à Paris et commercialise la marque Treez, des bracelets tendance qui contribuent à la reforestation. C'est une entreprise commerciale et écoresponsable : un bracelet vendu, c'est un arbre planté dans un projet de reforestation quelque part dans le monde. Elle évolue dans un marché de plus de 1,4 milliard d'euros. Malgré la concurrence (250 fabricants importateurs promotionnels dont $4 \%$ dits « Green »), elle se différencie des acteurs du secteur sur différents points: fabrication française, soutien à la reforestation, éco-conception et éco-innovation ${ }^{7}$. Son fondateur Alexis Krycève (A.K.) est particulièrement engagé socialement et au niveau environnemental et dispose d'une formation et d'une expérience professionnelle entrepreneuriales. La figure 1, illustre les principales caractéristiques de l'entreprise Gifts for change (Treez), telles que évaluées par les contributeurs de Wiseed.

Figure 1 - Principales caractéristiques de l'entreprise Gifts for change (Treez)

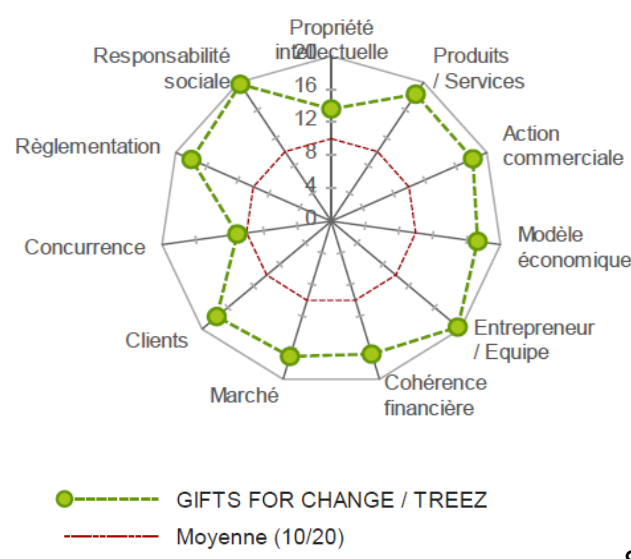

Source: $\underline{w w w . w i s e e d . c o m}$

L'ambition de notre travail empirique est de comprendre les motivations du recours de l'entreprise Gifts for change (Treez) au financement participatif rémunéré plutôt qu'au financement classique et d'expliquer son choix de faire appel successivement au crowdlending et à l'equity crowdfunding. Les avantages et les inconvénients de chaque forme de crowdfunding rémunéré seront mis en avant et une analyse comparative sera réalisée. En effet, l'entreprise Gifts for change (Treez) a effectué deux campagnes de collecte de fonds : la première en 2015 sous forme de prêt auprès de la plateforme Blue Bees et la seconde en 2016 sous forme de souscription d'actions auprès de la plateforme Wiseed. Ainsi, ce cas se prête bien à une analyse comparative entre ces deux types de financement surtout que faire appel aux deux formes de financement participatif rémunéré par une même entreprise reste aujourd'hui très rare.

Par ailleurs, l'entreprise Gifts for change a bien réussi son parcours de financement et son fondateur a su activer les bons dispositifs au bon moment. II convient de signaler que ce dernier a estimé que le crowdfunding sous forme de don pur n'était pas approprié au cas de son entreprise (commerciale / à but lucratif) et qu'au début

\footnotetext{
${ }^{7}$ Informations communiquées par le fondateur de Gifts for change (A.K.) et disponibles sur les pages des campagnes des deux levées de fonds de cette entreprise sur les sites de Wiseed et de Blue Bees.
} 
de l'activité de son entreprise, sa gamme de produit n'était pas assez large (un bracelet avec cinq déclinaisons) pour envisager une campagne sous forme de don avec contrepartie.

Les tableaux 1 et 2 représentent respectivement l'historique de financement de l'entreprise Gift for changes (Treez) depuis sa création et les caractéristiques des deux levées de fonds de financement participatif rémunéré.

Tableau 1 - Historique de financement de Gifts for change (Treez) depuis sa création

\begin{tabular}{|l|l|c|}
\hline Date & $\begin{array}{c}\text { Nature du } \\
\text { financement }\end{array}$ & $\begin{array}{c}\text { Montant du } \\
\text { financement }\end{array}$ \\
\hline 2014 & Apport personnel & $5000 €$ \\
\hline 2014 & Love money & $25000 €$ \\
\hline 2014 & Prêt d'honneur & $30000 €$ \\
\hline 2015 & Crowdlending & $33420 €$ \\
\hline 2016 & Equity crowdfunding & $250000 €$ \\
\hline 2016 & Business Angel & $100000 €$ \\
\hline
\end{tabular}

Tableau 2 - Caractéristiques des deux levées de fonds de financement participatif rémunéré de Gifts for change (Treez)

\begin{tabular}{|l|c|c|}
\cline { 2 - 3 } \multicolumn{1}{c|}{} & Crowdlending & Equity crowdfunding \\
\hline Date & 2015 & 2016 \\
\hline Montant & $33420 €$ & $250000 €$ \\
\hline Plateforme & Blue Bees & Wiseed \\
\hline Durée de la campagne & 70 jours & 3 mois \\
\hline Nombre d'investisseurs & 198 & 220 \\
\hline Rémunération des investisseurs & Taux fixe de 2\% & Variable \\
\hline Rémunération de la plateforme & $5 \%$ TTC du montant emprunté & $8 \%$ TTC du montant levé \\
\hline Horizon de l'investissement & $\begin{array}{c}\text { Remboursement en 3 ans ; } \\
\text { échéances semestrielles : }(4 \\
\text { échéances respectées au } \\
30 / 03 / 2017)\end{array}$ & $\begin{array}{c}\text { Entre 5 et 7 ans (sorties } \\
\text { envisageables : Cash out } \\
\text { avec entrée d'un nouvel } \\
\text { acteur de financement / } \\
\text { Rachat industriel }\end{array}$ \\
\hline
\end{tabular}

Comme signalé précédemment, les deux plateformes sur lesquels les campagnes ont été réalisées sont Blue Bees et Wiseed. Blue Bees est la première plateforme de financement participatif spécialisée dans l'agriculture et l'alimentation écologique. C'est une petite plateforme qui propose de financer les projets sous forme de dons avec ou sans contrepartie et sous forme de prêt. Elle a été créée en 2014 et a, depuis, financé 129 projets avec succès. Les montants levés sont de l'ordre de 2,5 millions d'euros dont presque la moitié est sous forme de prêt. Wiseed, créée en 2009, est un acteur historique de l'equity crowdfunding. En France, c'est le leader du secteur et la première plateforme à obtenir le statut de Conseillers en Investissement Participatif (CIP) en 2014 et l'agrément en tant que Prestataire de Services en

\footnotetext{
${ }^{8}$ En fonction de la performance de l'entreprise (société déficitaire au 31/12/2016).
} 
Investissement (PSI) de la part de l'autorité du contrôle prudentiel de résolution (ACPR) en 2016. La plateforme, bien qu'elle soit généraliste, se base sur un triptyque de mots clés : durable, responsable et performance. 175 projets ont été financés grâce à cette plateforme pour 79 millions d'euros de fonds levés. Le tableau 3 résume les principales caractéristiques de chaque plateforme ${ }^{9}$.

Tableau 3 - Principales caractéristiques des plateformes Blue Bees et Wiseed

\begin{tabular}{|l|c|c|}
\cline { 2 - 3 } \multicolumn{1}{c|}{} & Blue Bees & \\
\hline Spécialiste / généraliste & Spécialiste : Agriculture & Généraliste \\
\hline Statut & IFP & PSI \\
\hline Forme de financement & $\begin{array}{c}\text { Prêt et Dons (avec ou sans } \\
\text { contrepartie) }\end{array}$ & $\begin{array}{c}\text { Investissement (actions et } \\
\text { obligations) }\end{array}$ \\
\hline Montants investis & $2,6 \mathrm{M} €$ & $82 \mathrm{M} €$ dont $50,3 \%$ en actions \\
\hline Nombre de projets financés & 129 dont 50 en prêt & 179 dont 68 startups \\
\hline Montant moyen par projet & $50000 €$ & $322000 €$ (pour les startups) \\
\hline Ticket minimum de la & $20 €$ & $100 €$ \\
\hline $\begin{array}{l}\text { Commissions } \\
\text { plateforme }\end{array}$ & $5 \%$ TTC (pour le prêt) & Frais fixes +5 à $9 \%$ \\
\hline Sorties positives & $572000 €$ remboursés & 1 (plus-value de $+44 \%)$ \\
\hline Sorties négatives & 1 défaut de paiement & 9 \\
\hline
\end{tabular}

Nos sources de données sont multiples : d'abord, des données primaires grâce à des entretiens semi-directifs avec le fondateur de l'entreprise Alexis Krycève ainsi qu'avec des responsables des deux plateformes Blue Bees et Wiseed (respectivement Emmanuelle Paillat et Elodie Manthé). Les entretiens ont duré entre $50 \mathrm{mns}$ et $1 \mathrm{~h} 40$ et ont été retranscrits intégralement (67 pages au total). Ensuite, des données secondaires à travers une étude documentaire en mobilisant les informations disponibles sur les sites des plateformes sur lesquelles les campagnes ont été effectuées.

\section{Les motivations de l'entrepreneur pour se financer par le crowdfunding rémunéré}

Il ressort de notre analyse que différentes raisons peuvent être à l'origine du recours au financement participatif rémunéré. Les motivations de l'entrepreneur sont liées aux avantages du crowdfunding, d'une façon générale, mais également à certains atouts spécifiques au crowdlending et à l'equity crowdfunding.

D'abord, l'objectif premier est naturellement la levée de fonds. En effet, l'entreprise est à un stade où pour assurer son lancement, il est indispensable « d'obtenir du cash au départ »(A.K). À cette étape de la vie de l'entreprise, l'accès aux acteurs traditionnels du financement s'avère compliqué compte tenu du risque et du manque de visibilité de la start-up. Au-delà du refus éventuel des banques et des capitalrisqueurs, le porteur de projet peut lui-même s'estimer non éligible. L'autocensure est un comportement qu'on peut relever chez le porteur de projet étant données la nature des besoins à financer et la jeunesse du projet. D'une part, l'entrepreneur peut estimer que l'emprunt bancaire est plus adapté pour financer un investissement matériel (machines, locaux, équipements...) plutôt que le développement commercial, le BFR ou la communication qui représentent pourtant des dépenses

\footnotetext{
${ }^{9}$ Les données sur les plateformes correspondent aux données collectées en mai 2017.
} 
indispensables pour certains projets : "les banques ne savent pas financer...C'est très dur ou quasiment impossible pour des banques » (A.K). D'autre part, l'entrepreneur peut considérer que son projet est irrecevable en raison de la sélectivité du venture capital à ce stade d'amorçage "On était trop petit ; c'était trop tôt ; on a écarté le venture capital à l'époque...notre projet n'aurait jamais été accepté... ». II existe des critères objectifs qui font que les entreprises ne sont pas éligibles à un venture capital ou à un emprunt bancaire alors que n'importe quel projet peut être éligible au financement participatif. "Un fonds d'investissement va avoir des règles d'investissement beaucoup plus strictes que celles que nous avons » (Wiseed) ; “ ...on est plus souple sur certains critères... » (Blue Bees). En effet, le financement participatif se base notamment sur des critères subjectifs. Les codes du financement participatif, s'apparentent à l'aptitude à " embarquer une communauté " en leur " racontant une histoire " (A.K) ou à l'existence d'un réseau personnel étendu. Ils sont ainsi différents de ceux du financement classique uniquement basés sur la rentabilité et le risque de l'investissement. L'avantage du prêt par la foule est qu'il est accessible sans obligation de " montrer patte blanche en matière de solvabilité " (Blue Bees). Le crowdlending n'impose pas de garanties personnelles ou de cautions « contrairement à la banque, nous ne demandons pas à l'entrepreneur de garanties, ni d'hypothèque, ni de cautions...s'il les a nous les acceptons 》 (Blue Bees).

Ensuite, au-delà du levier de financement, le crowdfunding représente un bon levier de communication, particulièrement si le projet a une dimension grand public assez forte. Une campagne de crowdfunding est une opportunité de promotion participative bien que ce ne soit pas son objectif premier. L'avantage de ce mode de financement est par conséquent la visibilité qu'il confère aux clients potentiels «ils peuvent aussi bénéficier de la visibilité de la plateforme »; "... l'histoire de visibilité qui est quand même assez importante...” (Wiseed).

En outre, le financement par la foule permet de toucher un nombre important de personnes et fait appel à une dimension communautaire "Il va y avoir deux communautés qui vont se retrouver, les gens qui sont déjà dans la plateforme et qui font partie de la communauté de Wiseed et les gens que le dirigeant va lui-même amener sur la page du projet en ligne et donc là on va avoir la rencontre entre les deux... Donc il y a une dimension communautaire qui est très forte " (Wiseed). Le crowdfunding fait appel également à une dimension d'engagement "les gens qui financent ces projets viennent par engagement » (Blue Bees). II sollicite une forme de générosité des contributeurs. Ces derniers ont le sentiment de participer à "l'éclosion d'un projet » (A.K) et à l'aboutissement d'une idée. Cette dimension d'engagement est liée à la dimension entrepreneuriale puisque les investisseurs s'associent à l'aventure entrepreneuriale « les investisseurs ont envie de participer et de vivre un peu l'expérience par procuration" (Wiseed). Ainsi les projets qui bénéficient de ce levier « foule " ont une dimension émotionnelle ou innovatrice et un engagement éthique fort.

Enfin, le financement par crowdfunding rémunéré offre une souplesse et une rapidité d'implémentation qu'on ne retrouve pas dans le financement classique. Les start-ups sont, en effet, dans un milieu très concurrentiel qui leur exige une forte réactivité. Par conséquent, certains entrepreneurs en quête de rapidité s'orientent directement vers les plateformes de financement participatif rémunéré dont les dossiers sont plus simples à mettre en place et les réponses sont plus rapides. Alexis Krycève vente la 
simplicité de la mise en œuvre du dossier de prêt sur Blue Bees. Dans ce cas, l'entrepreneur, bien qu'il soit sollicité, ne s'éloigne pas trop de son activité quotidienne. La plateforme de prêt (ici Blue Bees) permet, en effet, de débloquer les fonds plus rapidement qu'une banque où le processus d'octroi de prêt est plus hiérarchisé " on débloque les fonds beaucoup plus rapidement en 1 mois vous pouvez avoir $50000 €$ sur Blue bees »; " au niveau des banques, il' y a des échelons, c'est hiérarchisé, il y a besoin de validation et de sur-validation". Sur Wiseed, la durée moyenne d'une collecte est de 75 jours pour les start-ups. La campagne de Gifts for Change a duré trois mois. Wiseed estime que la durée de toute la démarche est moins longue que celle d'un venture capital et que les équipes de la plateforme essaient d'écourter au mieux la phase de sélection en apportant une aide et des conseils aux porteurs de projets ; "en terme de temps, on est très compétitif par rapport à des fonds d'investissement, par exemple » (Wiseed).

Par ailleurs, le financement par la foule ne présente pas de risque particulier pour l'entrepreneur. Néanmoins, il existe une appréhension de la part des entrepreneurs concernant la capacité à collecter la somme souhaitée «...il y'avait peu de risque pour moi... »; " J'estimais que la plus grande crainte c'est de ne pas pouvoir arriver au bout de ma collecte" (A.K). Les plateformes jouent ici un rôle important d'encadrement et de conseil pour les entrepreneurs. Elles peuvent être flexibles et « s'arrangent » pour que les campagnes soient réussies soit en réduisant l'objectif soit en prolongeant la durée de la campagne.

\section{Le choix de la forme de financement participatif : crowdlending vs. crowdequity}

Le crowdfunding sous forme de don sans contrepartie n'est pas adapté aux projets entrepreneuriaux. II convient plutôt aux associations, aux projets à but non lucratif ou à certains projets artistiques ou culturels. Le don avec contrepartie, bien qu'il permette d'utiliser pleinement le ressort communautaire dans sa dimension de participation collaborative et de créativité afin de préfinancer de nouveaux produits, il nécessite que l'entreprise dispose ou puisse développer des contreparties spécifiques avec une large gamme de produits. Ainsi, le crowdfunding rémunéré représente ici une bonne alternative de financement. II reste à déterminer quelle forme de financement participatif rémunéré est la plus adaptée selon le projet.

Le choix entre le crowdlending et l'equity crowdfunding ne se pose pas forcément au même stade de développement de l'entreprise ni au même niveau de maturité. Ce choix dépend simultanément du montant des fonds à lever et de la nature des besoins à financer.

Lorsque le besoin de financement est important mais porte sur des actifs intangibles, de la recherche et développement, de la communication ou encore l'investissement dans l'humain, l'equity crowdfunding serait plus approprié «...on ne financera pas beaucoup le BFR... L'idée est quand même de financer des dépenses qui permettront de créer de la valeur au sein de l'entité juridique que représente la startup; donc nous allons financer du développement commercial, de la $R \& D$, des embauches...» (Wiseed). Le crowdlending, en revanche, serait la solution si les montants à lever sont faibles ou si l'activité de l'entreprise requiert des investissements lourds en BFR, par exemple. . (Treez a levé $33420 €$ dans le cadre de la campagne de prêt avec la plateforme Blue Bees). Dans le premier cas, le 
crowdlending serait un tremplin permettant de solliciter la communauté de contributeurs afin de collecter des petites sommes nécessaires à des besoins ponctuels. Dans le deuxième cas, le crowdlending répondrait aux difficultés de certaines entreprises à se financer auprès des banques traditionnelles à cause de la nature du besoin à financer ou de leur faible solvabilité "On finance plutôt du BFR, parce que les banques sont réticentes à financer » (Blue Bees). Si le projet est déjà solvable ou si l'entrepreneur dispose des garanties nécessaires, le recours à l'emprunt bancaire peut s'avérer plus simple. En effet, faire appel aux contributeurs pour emprunter des montants élevés nécessite un gros effort de visibilité et pourrait user la communauté à force de la solliciter. Dans ce contexte, la contrepartie financière devient alors la principale motivation des crowdfundeurs. Par ailleurs, l'equity crowdfunding permet de lever des fonds plus conséquents (par exemple le montant de la collecte de l'entreprise Treez s'élève à $250000 €$ et la collecte moyenne pour les start-ups chez Wiseed est de $315600 €)$. Aussi le crowdequity peut être envisagé dans le cadre d'une levée de fonds plus conséquente conjointement avec les autres acteurs du financement. Le co-investissement est une pratique courante dans le crowdequity, contrairement au crowdlending « Des fois les porteurs de projet cherchent des montants tellement importants qu'un seul acteur ne suffirait pas... » (Wiseed). Pour ces raisons, l'equity crowdfunding représente en général la dernière étape de financement participatif.

Selon Alexis Krycève, l'equity crowdfunding est une sorte de financement hybride entre un venture capital et la foule/le grand public. II s'agit d'une mutualisation de capacités d'investissement plus restreintes d'un grand nombre de personnes dans un format qui ressemble à un venture capital. L'equity crowdfunding permet à la fois à une entreprise de trouver les investissements conséquents que peuvent nécessiter son développement, tout en se dotant d'un actionnariat qui partage ses valeurs.

L'equity crowdfunding nécessite, en revanche, un effort plus soutenu de la part de l'entrepreneur. La démarche requiert davantage de temps et d'énergie. Une double compétence marketing et financière est souhaitable. L'entrepreneur doit convaincre à la fois la foule, responsable de la décision de financement, et les spécialistes de la plateforme qui se chargent de la due diligence et de la détermination du prix. L'horizon de la sortie du capital est différent dans les deux catégories de financement participatif rémunéré. Les plateformes de prêt disposent d'un coussin de financement grâce au remboursement d'une partie des prêts en cours qui peut être utilisé pour financer des nouveaux projets. Les plateformes de financement sous forme d'actions ne bénéficient pas du même dispositif (Wiseed par exemple ne compte qu'une seule sortie positive à ce jour). Par conséquent, les porteurs de projet ainsi que les plateformes sont obligés de solliciter de nouveaux investisseurs et de doubler d'effort en termes de communication "Sur le prêt il n'y a pas besoin de beaucoup communiquer maintenant.... Ça marche tout seul, puisque l'argent tourne... » (Blue Bees). 
Le tableau 4 résume les principales différences entre le crowdlending et l'equity crowdfunding.

Tableau 4 - Principales différences entre le crowdlending et l'equity crowdfunding

\begin{tabular}{|l|c|c|}
\cline { 2 - 3 } \multicolumn{1}{c|}{} & Crowdlending & Equity crowdfunding \\
\hline Nature du financement & Dettes & Fonds propres \\
\hline Montants levés & Faibles / moyens & Elevés / moyens \\
\hline Principaux besoins à financer & $\begin{array}{c}\text { BFR; développement } \\
\text { commercial ; recrutement }\end{array}$ & $\begin{array}{c}\text { R\&D ; Développement } \\
\text { commercial ; développement à } \\
\text { l'international }\end{array}$ \\
\hline Stade de développement & $\begin{array}{c}\text { Amorçage (Avant le } \\
\text { crowdequity) }\end{array}$ & $\begin{array}{c}\text { Amorçage (stade plus } \\
\text { développé) }\end{array}$ \\
\hline $\begin{array}{l}\text { Durée moyenne de } \\
\text { l'investissement }\end{array}$ & 3 ans (maximum 7 ans) & Entre 5 et 7 ans au minimum \\
\hline Intervention dans la gestion & Passive & Passive \\
\hline Démarche / efforts & $\begin{array}{c}\text { Démarche simple / efforts } \\
\text { modérés }\end{array}$ & $\begin{array}{c}\text { Démarche proactive / Efforts } \\
\text { conséquents (communication ; } \\
\text { due diligence) }\end{array}$ \\
\hline Risque de dilution du capital & Non & Oui (modéré) \\
\hline $\begin{array}{l}\text { Risque de perte de contrôle ou } \\
\text { flexibilité }\end{array}$ & Non & Non \\
\hline
\end{tabular}

\section{Conseils à suivre pour réussir son financement par la foule d'investisseurs}

Le crowdfunding ne représente pas la meilleure alternative de financement dans l'absolu. "Ce sont certains dispositifs de certaines plateformes qui sont adaptés à certains projets à un certain stade de développement de l'entreprise ". Alexis Krycève évoque l'importance de savoir embarquer une communauté avec soi. Les projets ayant une forte dimension grand public sont plus éligibles au financement via le crowdfunding. La notion d'éthique est compatible avec ce mode d'allocation de ressources car la foule contribue à l'éclosion d'un projet avec des vertus sociales et environnementales. II ressort des entretiens que l'effet communauté, l'adhésion à des valeurs et des principes spécifiques, est cohérent avec le financement participatif, même rémunéré. Le financement participatif permet aux contributeurs mais également aux entreprises de s'engager pour le changement à travers des actions sociales et environnementales (communication par l'objet ou pas). D'où l'intérêt de choisir une plateforme spécialisée qui a une communauté engagée si le projet est caractérisé par une dimension sociale ou éthique forte. L'objectif de certaines plateformes spécialisées va au-delà des critères d'investissement habituels. Par exemple, la communauté de Blue Bees est composée de personnes très engagées. Pour cette plateforme, le collaboratif est un outil mais l'objectif est de changer l'agriculture. II est donc très important, quand on s'adresse à une communauté engagée, de montrer l'utilité sociale du projet et d'insister sur son sérieux. Les entrepreneurs peuvent faire appel aux plateformes éthiques si leur projet est d'intérêt général avec un but de sensibilisation, même s'il n'a pas forcément de modèle économique. Dans ce type de plateforme, les facteurs de succès sont étroitement liés à l'émotion que crée le projet et à la manière dont l'entrepreneur communique et présente le projet. Les contributeurs ne sont donc pas à la recherche de rentabilité même s'ils investissent dans des prêts rémunérés. Si le projet n'a pas comme objectif la création de valeur sociale ou environnementale, il 
existe des plateformes de prêt plus spéculatives mais le coût de financement dans ce cas peut s'avérer plus élevé puisque le critère rentabilité-risque serait prépondérant «... on a des entrepreneurs qui nous ont disent maintenant on a des taux zéro quasiment auprès des banques, ils se disent qu'est-ce qu'on va aller s'embêter avec le crowdlending... »

Le financement à travers l'equity crowdfunding nécessite une démarche encore plus proactive de la part du porteur de projet. L'entrepreneur doit bien choisir la plateforme et analyser les caractéristiques et les conditions de la concurrence avant de se lancer dans la campagne de collecte de fonds. II faut trouver la plateforme qui assure le plus de complémentarité avec le porteur de projet. Les mécanismes d'accompagnement et de suivi de l'entreprise dans les différentes phases de la levée de fonds (pré-investissement et post-investissement) sont des paramètres importants à prendre en considération. Le sérieux et la réputation des plateformes de crowdfunding est également un critère à privilégier. Ces plateformes ont un business model se basant principalement sur des commissions ou des frais de gestion sur les fonds collectés. Blue Bees prend $8 \%$ TTC sur le don et $5 \%$ TTC sur le prêt; Wiseed facture 5 à $9 \%$ sur le montant levé. Ces frais varient en fonction des plateformes. Outre le partage de vertus sociaux, environnementaux ou éthiques communs avec les projets, les plateformes s'investissent par conséquent parce qu'elles ne sont rémunérées que lorsque la campagne aboutit.

Une fois la plateforme choisie, il faut franchir la phase de sélection. Les entrepreneurs ne doivent pas sous-estimer le taux de rejet des plateformes de prêt ou de participation en capital. Certes, ces dernières sont moins sélectives que les acteurs établis du système financier " un fonds d'investissement va avoir des règles d'investissement beaucoup plus strictes que celles que nous on a " (Wiseed), mais elles le sont néanmoins. Le taux de sélection positive est par exemple de $3 \%$ pour les start-ups chez Wiseed " on a quasiment les mêmes taux de sélection que dans un fonds d'investissement don ce n'est pas parce qu'ils viennent nous voir qu'on va les sélectionner». La directrice de Blue Bees annonce « on ne finance pas tout le monde, on ne prend pas des risques inconsidérés ... ". La sélectivité est moindre au niveau de la taille minimale des levées de fonds et sur certains critères d'évaluation. L'implication et la motivation du porteur du projet peuvent être considérées comme un paramètre atténuant le risque du projet. Toutefois, ces nouveaux acteurs de financement se basent également sur des méthodes traditionnelles comme l'analyse de crédit à travers des notations financières pour le prêt ou l'analyse financière par les ratios pour le financement par actions. Les start-ups les plus éligibles sont celles qui ont déjà entamé leur activité (des clients et un chiffre d'affaires) et qui souhaitent accélérer leur développement. L'equity crowdfunding peut être approprié dans la phase d'amorçage mais les entreprises appartenant à certains secteurs, comme la biotech par exemple, sont les plus éligibles dans ce cadre. En outre, une première allocation des ressources grâce à la love money et à l'autofinancement augmente les chances d'être choisi par les plateformes. Bien que le processus soit rapide, l'étape de pré-investissement peut s'avérer longue pour l'entrepreneur à cause du nombre d'étapes de sélection et de la phase de financement puisqu'il ne faut pas négliger le temps de la collecte «...il y a un temps de collecte...là où un fonds d'investissement peut mettre du temps à dire oui mais quand qu'il a dit oui, les fonds sont versés en 15 jours, là c'est l'inverse, d'abord devoir collecter auprès des particuliers l'argent puisqu'on ne l'a pas en stock » (Wiseed)

Par ailleurs, la négociation des paramètres de la levée de fonds est primordiale. II est par conséquent nécessaire de : 1- fixer de façon raisonnable et fondée le montant à 
lever, 2- de s'assurer de la valorisation de l'entreprise puisque ça détermine notamment le pourcentage de contrôle des nouveaux actionnaires et impacte le pourcentage de dilution et 3 - de faire attentions aux clauses dans le pacte d'actionnaires ou dans le contrat d'emprunt.

Enfin, le porteur de projet doit être conscient que le recours à la foule a également des inconvénients auxquels il devrait porter une attention particulière et essayer d'en minimiser les effets. Blue Bees évoque l'appréhension de décevoir un nombre important d'investisseurs. Réussir à financer un projet via le crowdfunding est un signe d'encouragement, de soutien et de confiance pour l'entrepreneur. La responsabilité de ce dernier s'en trouve alors plus importante et la pression psychologique qu'il supporte également. Les responsables des plateformes interrogés avancent qu'il est beaucoup plus facile de décevoir un banquier ou un fonds d'investissement qu'un nombre important de contributeurs qui ont fait confiance au porteur de projet. Par ailleurs, commencer une campagne de crowdfunding nécessite beaucoup d'implication de la part des porteurs de projets. La durée de collecte des fonds est certes réduite mais elle nécessite une mobilisation continue assez forte. Alexis Krycève estime que « c'est assez chronophage et ça doit être considéré comme un projet à part entière de l'année de développement ». Une première campagne de financement participatif nécessite par conséquent une implication personnelle forte.

\section{Conclusion}

Au-delà des avantages connus du crowdfunding, cet article contribue à la fois à la recherche sur les spécificités du crowdfunding spéculatif et la comparaison de ses deux composantes : l'equity crowdfunding et le crowdlending.

L'étude de cas montre que le financement participatif rémunéré est un moyen de financement plus flexible que le financement traditionnel et qu'il représente une solution de financement appropriée sous quelques conditions. II apparaît, par ailleurs, comme un levier permettant d'accéder avec plus de légitimité aux acteurs classiques de financement.

Le crowdfunding n'est pas la meilleure alternative de financement dans l'absolu. "Ce sont certains dispositifs de certaines plateformes qui sont adaptés à certains projets à un certain stade de développement de l'entreprise ». D'abord, il s'agit d'un mode de financement qui est à la fois cohérent avec la dimension sociale, éthique ou innovatrice des projets ou start-ups à financer et avec l'environnement concurrentiel à forte croissance dans lequel ils évoluent. Avec le crowdfunding rémunéré, le porteur de projet, s'affranchit de l'autocensure relative à l'éligibilité de son projet au financement par les acteurs établis du secteur financier. Aussi, l'entrepreneur acquiert une légitimité à financer son projet par la foule sans se limiter à la générosité des contributeurs ni être obligé de développer une contrepartie spécifique. Toutefois, le financement participatif rémunéré ne représente pas la solution miracle au financement des TPE et des PME. Les plateformes demeurent sélectives bien que certains de leurs critères soient subjectifs et offrent, par conséquent, plus de souplesse.

En outre, notre étude montre que le choix entre le crowdlending et l'equity crowdfunding ne se pose pas forcément au même stade de développement de l'entreprise. Ce choix dépend à la fois du montant des fonds à lever et de la nature des besoins à financer. Les deux formes de financement participatif rémunéré ne requièrent pas le même effort ni la même implication de la part du porteur de projet. 
Enfin, en matière de recherche sur le financement participatif, notre étude complète les réflexions sur la pertinence de ce type de financement en mettant en garde les entrepreneurs contre ses limites et en leur proposant la démarche à suivre afin de maximiser la chance de réussir leurs campagnes.

Les limites de cette recherche se rattachent principalement à la limite intrinsèque de la méthodologie utilisée et à l'évolution rapide du secteur du crowdfunding. Aussi, afin de compléter nos recommandations et généraliser nos résultats nous sommes dans un processus continu de collecte d'informations et d'entretiens avec les spécialistes du domaine (responsables de différentes plateformes) ainsi qu'à la recherche de nouveaux témoignages et expériences entrepreneuriales. 


\section{Bibliographie}

Agrawal A., Catalini C., Goldfab A. (2011), The geography of crowdfunding, Working Paper, University of Toronto.

Belanovà K. (2015), Crowdfunding: an innovative financial source of small and medium sized enterprises, Young Economists Journal, Vol. 12. №24. pp. 137-146.

Belleflamme P., Lambert T., Schwienbacher A. (2014), Crowdfunding: Tapping the right crowd, Journal of Business Venturing, Vol.29, n5, pp. 585-609.

Bendriss J., Lavayssière B., Tilden M. (2014), Le financement des PME en France : un contexte particulier, favorable aux innovations, Revue d'Economie Financière, Vol. 2, № 114, pp. 241-254.

Bertrand C., Jakubowski B. (2016), Le fric, c'est chic : panorama du crowdfunding en 2016, Annales des Mines - Réalités industrielles, Vol. 1, pp. 38-43.

Bessière V. et Stéphany E. (2014), "Le financement par crowdfunding: Quelles spécificités pour l'évaluation des entreprises? "Revue Française de Gestion, Vol.40, № 242, p. 149-161.

Bessière V. et Stéphany E. (2015), " Financement et gouvernance des start-ups en equitycrowdfunding » Finance Contrôle Stratégie, Vol.18, № 4, p. 1-26.

Blanchard A., Sabuncu E. (2016), Avis du comité consultatif de déontologie et d'éthique de l'IRD sur le crowdfunding: intérêts et limites, Natures Sciences Sociétés, №2, Vol. 24, pp. 154-159.

Cai, S., Lin, X., Xu, D., Fu, X. (2016), Judging online peer-to-peer lending behavior: A comparison of first-time and repeated borrowing requests. Information \& Management, 53(7), pp. 857-867.

Cailloux J., landier A. et Plantin G. (2014), Crédit aux PME : des mesures ciblées pour des difficultés ciblées, Notes du conseil d'analyse économique, Vol. $8, n^{\circ} 18$, pp.1-12.

Emekter, R., Tu, Y., Jirasakuldech, B., Lu, M. (2015), Evaluating credit risk and loan performance in online Peer-to-Peer lending. Applied Economics, Vol. 47, n¹, pp. 5470.

Fasshauer I., (2016) «Contrôler par la foule ? Le cas des plateformes d'equitycrowdfunding ", Revue Française de Gestion, n²59, pp. 51-68.

Fliche O., Castanet C., Saidani C., Delannoy A. (2016), La régulation des plateformes de financement participatif (crowdfunding) en France, Annales des Mines - Réalités industrielles, № 1, pp. 57-60.

Gajda O., Walton J. (2013), Review of crowdfunding for development initiatives. IMC Worldwide for Evidence on Demand.

Gerber, E. M., Hui, J. S., \& Kuo, P. Y. (2012), Crowdfunding: Why people are motivated to post and fund projects on crowdfunding platforms. In Proceedings of the 
International Workshop on Design, Influence, and Social Technologies: Techniques, Impacts and Ethics, Vol. 2.

Girard C. et Deffains-Crapsky C. (2016), Les mécanismes de gouvernance disciplinaire et cognitifs en Equity Crowdfunding: Le cas de la France ", Finance Contrôle Stratégie, vol.19, n 3, pp. 1-15.

Giudici G., Nava R., Lamastra, C. R., Verecondo C. (2012). Crowdfunding: The New Frontier for Financing Entrepreneurship?, Working paper, SSRN Papers.

Golić Z. (2014), Advantages of Crowdfunding as an alternative source of financing as an alternative source of financing of small and medium-sized enterprises. Proceedings of the Faculty of Economics in East Sarajevo, $N^{\circ}$ 8, pp. 39-48.

Gonzalez, L., Loureiro, Y. K. (2014), When can a photo increase credit? The impact of lender and borrower profiles on online peer-to-peer loans, Journal of Behavioral and Experimental Finance, Vol. 2, pp. 44-58.

Herzenstein, M., Sonenshein, S., Dholakia, U. M. (2011). Tell me a good story and I may lend you money: The role of narratives in peer-to-peer lending decisions. Journal of Marketing Research, Vol. 48, pp. 138-S149.

Kettani G. et Villemeur A. (2012), Le capital-risque : un financement efficace de l'innovation sur le long terme, Revue d'Economie Financière, Vol. 108, № 4, pp. 91 104.

Kremp E., Piot C. (2014), Le ralentissement du crédit bancaire aux PME en France, Revue d'Economie Financière, Vol. 114, № 2, pp. 91-103.

Lambert, T., Schwienbacher, A. (2010), An Empirical Analysis of Crowdfunding, Working paper, Louvain-la-Neuve, Louvain School of Management, Catholic University of Louvain.

Larrimore, L., Jiang, L., Larrimore, J., Markowitz, D., Gorski, S. (2011), Peer to peer lending: The relationship between language features, trustworthiness, and persuasion success. Journal of Applied Communication Research, Vol. 39, n¹, pp. 19-37.

Lesur N. (2015), Les défis posés par le crowdfunding, Revue d'économie Financière, $\mathrm{n}^{\circ} 118$, p. 103-112.

Lesur N. (2016), Crowdfunding: financement de complément, ou de rupture ? Annales des Mines - Réalités industrielles, Vol. 1, pp. 8-11.

Maier E. (2016), Supply and demand on crowdlending platforms: connecting small and medium-sizes enterprises borrowers and consumers investors, Journal of Retailing and Consumer Services, $n^{\circ}$ 33, pp. 143-153.

Mahieux X. (2012), Le private equity américain après la crise : reconfiguration et maturité, Revue d'Economie Financière, Vol. 105, № 1, pp. 185-203.

Mollick E. (2014), The dynamics of crowdfunding: An exploratory study, Journal of Business Venturing, Vol. 29, pp.1-16.

O'Reilly T. (2005), «What's web2.0 », disponible à http://www.oreilly.com. 
Stake, R. E. (1995). The art of case study research. Thousand Oaks, CA: SAGE Publications.

Sviokla, J. (2009). Breakthrough ideas: forget Citibank - borrow from Bob. Harvard Business Review, Vol. 87, №2, pp. 19-40.

Valanciene, L., Sima J. (2013), Valuation of Crowdfunding: Benefits and drawbacks, Economics and Management, Vol. 18, n¹, pp. 39-48.

Vulkan N., Asterbo T., Sierra M.F. (2016), Equity crowdfunding : A new phenomena, Journal Of Business Venturing Insights, Vol. 5, pp. 37-49.

Yin, R. K. (1993). Applications of case study research. Applied Social Research Series, Vol. 34. London: Sage.

Yin, R. K. (2014), Case study research: Design and Methods, London: Sage publications.

Yum, H., Lee, B., Chae, M. (2012), From the wisdom of crowds to my own judgment in microfinance through online peer-to-peer lending platforms, Electronic Commerce Research and Applications, Vol. 11, n5, pp. 469-483. 